\section{Miradas locales y globales en la construcción de los barrios migrantes coreanos en Latinoamérica. Los casos de Baek-ku en Buenos Aires y Korea Town en Guatemala}

Matías Benítez ${ }^{(1)}$

Resumen: En este artículo nos proponemos realizar un análisis histórico-comparativo de la inmigración coreana a Latinoamérica a partir de los casos de Argentina y Guatemala; tomando como un eje transversal de dichos procesos de constitución comunitaria la construcción de los barrios migrantes. De esta manera buscaremos identificar y describir cuáles fueron los factores principales que incidieron en el asentamiento de los primeros coreanos migrantes y la conformación de las comunidades; y además cuáles son las dinámicas que actualmente atraviesan a las mismas tanto en Buenos Aires como en la Ciudad de Guatemala.

Palabras clave: migraciones internacionales - barrios migrantes - diáspora - Corea - Latinoamérica.

[Resúmenes en inglés y portugués en la página 134]

(1) Sociólogo egresado de la Universidad de Buenos Aires. Miembro del Instituto de Investigaciones Gino Germani dependiente de la Facultad de Ciencias Sociales de la UBA. Integrante del Centro de Estudios Coreanos de la Universidad Nacional de La Plata (CECOR).

\title{
Introducción
}

En el siguiente trabajo partimos de la consideración en torno a que el fenómeno demográfico de las migraciones internacionales se encuentra atravesado por una complejidad de procesos sociales y dimensiones culturales que han generado cambios profundos en las sociedades contemporáneas. En este sentido, hay autores que sostienen que “(...) en un mundo globalizado la lógica de los espacios muta y se evidencian simultaneidad de los procesos sociales y culturales de lugares distantes" (Cosiansi, Mera, 2015, p. 291). A partir de allí consideramos pertinente realizar una indagación en donde se aborden a partir de un enfoque histórico-comparativo cómo se ha producido la inmigración coreana a Latinoamérica a partir de los casos de Argentina y Guatemala; tomando como un eje 
transversal de dichos procesos de constitución comunitaria la construcción de los barrios migrantes. De esta manera, buscaremos identificar y describir cuáles fueron los factores principales que incidieron en el asentamiento de los primeros coreanos migrantes y la conformación de las comunidades; y además cuáles son las dinámicas que actualmente atraviesan a las mismas tanto en Buenos Aires como en la Ciudad de Guatemala. Si acudimos a los datos provistos por el Ministerio de Asuntos Exteriores de la República de Corea se observa que las tres comunidades coreanas más pobladas y antiguas (Brasil, Argentina y México) se mantienen prácticamente estancadas desde hace más de 10 años. En el caso de Argentina desde 2007 se mantiene en aproximadamente 22 mil habitantes. Por otro lado en Guatemala (una de las comunidades de instalación más reciente en donde empezó a darse el asentamiento de migrantes coreanos en 1985, 20 años después que Argentina) se observa un crecimiento poblacional que puede registrarse a partir de 1997 cuando eran sólo 2 mil habitantes hasta 2013 cuando llegó a tener 12.900 habitantes. Esto da cuenta de un dinamismo que no han tenido otras comunidades de la región. Si bien hay que aclarar que en el caso de Argentina la crisis económica y social que estalló en el año 2001 generó una emigración masiva de coreanos del país que dividieron sus destinos entre Estados Unidos, Canadá, México y Corea del Sur mayoritariamente (Mera, 2009). A partir de 2003 parte de aquellos emigrados retornó al país.

Para poder adentrarnos al estudio de estos dos casos que consideramos representativos de la diáspora coreana en América Latina, nos basaremos en los trabajos referidos a la inmigración coreana disponibles tanto en las bibliotecas como en buscadores académicos. Para el caso de inmigración coreana en Argentina, podemos identificar como principales antecedentes los trabajos de Carolina Mera entre los que se destaca el libro La inmigración coreana en Buenos Aires: multiculturalismo en el espacio urbano (1998) que es una primera aproximación al tema desde una perspectiva sociológica. En lo que refiere a un enfoque de tipo antropológico, se puede mencionar el trabajo de Corina Courtis Construcción de Alteridades: Discursos sobre la inmigración coreana en Buenos Aires (2000) que enfatiza en los elementos socio-lingüísticos y el de Mirtha Bialogorski. La presencia coreana en la Argentina: La construcción simbólica de una experiencia migratoria" (1999) entre otros. Al respecto a Guatemala el acceso a publicaciones sobre la temática es mucho más exiguo. Por lo que en consecuencia además de recurrir a los trabajos disponibles (que refieren en su mayoría a la llegada de capitales surcoreanos para invertir en maquilas durante los 80 's) relevaremos medios periodísticos y distintas fuentes gubernamentales (ministerios y embajadas) que nos permitan darle una mayor densidad a la caracterización de la comunidad coreana de Guatemala residente en la Capital. En este sentido habrá especial énfasis en el relevamiento (considerando el planteo principal del trabajo) de aquellas cuestiones ligadas al proyecto urbanístico Korea Town desde el cual estableceremos un contrapunto con Baek-ku, el barrio coreano de Buenos Aires.

En consideración de lo antedicho, desarrollaremos el planteo de este trabajo en tres secciones subsiguientes. En primer lugar ofreceremos un apartado conceptual donde problematizaremos el concepto de diáspora aplicado en el estudio de las comunidades coreanas. En el mismo concepto; el barrio étnico constituye uno de los elementos centrales para la construcción de identidades migrantes en el marco de la globalización. En la segunda sección, dividida en dos, desenvolveremos la perspectiva planteada abocándonos al análisis 
de la construcción de los barrios coreanos tanto de Buenos Aires (Baek-ku) como el de Guatemala (Korea Town). Finalmente, concluiremos destacando los principales contrastes y afinidades entre ambos casos. A partir de lo cual reflexionaremos en torno a las limitaciones a superar y potencialidades a aprovechar con el fin de afianzar la interculturalidad entendida como indicador de reciprocidad y tolerancia entre identidades diversas que se basa en el respeto a la experiencia de la diferencia del otro.

\section{Diáspora coreana y barrios migrantes en Latinoamérica Precisiones conceptuales}

Para el año 2019 los datos oficiales provistos por el Ministerio de Asuntos Exteriores de Corea del Sur muestran que habitan en Latinoamérica más de 100 mil coreanos ${ }^{1}$. Más allá de la entrada de 1033 trabajadores coreanos a México en 1905 (Kim, 2005) y de 62 militares norcoreanos prisioneros de guerra en 1955 (Mera, 2008; Kim, 2012, p. 115) divididos entre Argentina y Brasil (12 y 50 respectivamente), la migración coreana en gran escala a América Latina comenzó oficialmente a mediados de los 60 's. Esto se debió a las políticas de emigración planteadas por el Estado surcoreano que estuvieron relacionadas con los cambios que se produjeron en la península durante esa década. En este sentido, los principales factores que explican la promoción de las políticas de emigración remiten al crecimiento demográfico y la situación política, tanto interna como intercoreana (Mera, 2009). Tengamos en cuenta el aumento sustancial de población que se generó en el sur de Corea con los 2,3 millones de coreanos que regresaron al país al finalizar la Segunda Guerra Mundial y por el millón de norcoreanos que se refugiaron en el sur antes de estallar la guerra en 1950. Además de la mejora en la calidad de vida que se generó posteriormente a causa de las nuevas técnicas médicas importadas de Occidente. Cuestión por la cual se implementaron programas de planificación familiar y de control de la natalidad. A lo que se le suma el intento por bajar la tasa de desempleo y adquirir nuevas tecnologías para la producción industrial. Por su parte debe considerarse también la política interna represiva de los gobiernos militares surcoreanos, la situación de inestabilidad con Corea del Norte y la propensión a emigrar de la población cristiana, en especial la norcoreana que había adoptado ese credo (Radulovich, 2012, p. 278).

En este contexto, países latinoamericanos como Argentina, Bolivia, Brasil y Paraguay comenzaron a aceptar inmigrantes coreanos durante la década de 1960 para resolver la escasez de mano de obra agrícola. Sin embargo, al poco tiempo la mayor parte de aquellos migrantes se retiraron de las zonas rurales y se trasladaron a las ciudades. Esto se dio porque en algunos casos las tierras que se les habían asignado para las colonias agrícolas no estaban en condiciones para ser explotadas, y en otros porque el gobierno surcoreano no los había apoyado financieramente (Jin, 2017, p. 67). En suma, a los problemas de infraestructura este éxodo se puede explicar también por los antecedentes socioeconómicos de los migrantes, muchos de los cuales provenían de la clase media industrial, comercial y profesional y que nunca antes habían practicado actividades agrícolas ni rurales (Mera, 2008).Incluso aquellos inmigrantes que al principio intentaron dedicarse a la agricultura 
se dieron cuenta de que no era un negocio rentable y que a menudo estaba sujeto a importantes fluctuaciones de precios.

Una vez reasentados en la ciudad los migrantes coreanos buscaron actividades alternativas como estrategia de supervivencia. Las principales estuvieron relacionadas con el sector comercial y textil. La elección de esta actividad se justifica dado que son trabajos para las cuales no son necesarias importantes sumas de capital inicial, no se necesita de un gran conocimiento previo, ni se requieren competencias lingüísticas (Mera, 2009). En principio los migrantes realizaron trabajos de bordado y costura para negocios con dueños de la colectividad judía y posteriormente montaron establecimientos propios a partir del capital y experiencia acumulados (Mera, 1998; Bialogorsky, 2004). Esto generó un crecimiento económico de las comunidades que devino en la expansión de los negocios en todos los sectores de la fabricación de prendas de vestir, desde el diseño hasta el embalaje del producto final. Además, algunos coreanos comenzaron a operar tiendas de ropa al por menor $\mathrm{y}$ al por mayor. Otros inmigrantes coreanos incluso comenzaron participar en una gama más amplia de negocios relacionados con la indumentaria, por ejemplo en la importación de maquinaria textil (Jin, 2017).

Es en este contexto de instalación de los migrantes coreanos en las principales ciudades latinoamericanas donde se sentaría las bases para el proceso de construcción de espacios de la diáspora del que se puede dar cuenta por medio del análisis de las comunidades coreanas de la región. Ahora bien, para efectuar esta indagación nos basaremos en la perspectiva de Dufoix que define a las diásporas modernas como “... grupos étnicos minoritarios, producto de la migración, los cuales residen y actúan en los países receptores, a la vez que mantienen fuertes lazos afectivos y materiales con su país de origen, su patria" (2008, p. 23 en Mera, 2009). Es a partir de este concepto que nos permitimos reflexionar acerca de las formas de existencia que adoptan las comunidades migrantes en la etapa actual de la globalización. Esta se caracteriza por un incremento de la pluralidad étnica y cultural de las ciudades, que por medio de las migraciones nacionales a internacionales, promueven la interpenetración de poblaciones y formas de vida disímiles en el espacio de las principales áreas metropolitanas del planeta. Nos encontramos insertos en la trama contemporánea donde "lo global se localiza, de forma socialmente segmentada y especialmente segregada, mediante los desplazamientos humanos provocados por la desaparición de viejas formas productivas y la creación de nuevos centros de actividad" (Sassone y Mera, 2007, p. 10). Por lo que entonces no será como en la diáspora clásica el fundamento central la expulsión por un hecho traumático sino

(...) el sentido de pertenencia que se refiera a una identidad nacional, cultura o religión, que si bien es articulada por un Estado-Nación o cultura nacional, pone en juego formas de construir identidades y de mantener una unidad de la misma en el plano transnacional. El desarraigo del territorio de origen, inherente a toda diáspora, supone la necesidad de una memoria colectiva que posibilite elaborar el cambio desde lo local-global (Mera, 2011, p. 4).

El fenómeno diaspórico tiene entonces un carácter transnacional y se compone de una serie de prácticas significantes en donde se incorporan las estructuras sociales del país re- 
ceptor a la par que se genera una adhesión étnica al país de origen. El mismo da inicio con una ruptura con el país de origen (no catastrófica en el caso coreano), donde se constituyó su cultura y su identidad. Continúa con el proceso de instalación en la sociedad receptora, y finalmente llega a su punto culmine con la extensión de la red trasnacional. Durante el proceso se generan y articulan las formas sociales que son el centro de la cadena migratoria. En este caso las principales son la familia, las iglesias y las asociaciones de migrantes (las dos últimas además funcionan como instancias institucionales de la comunidad). La familia constituye la red de sociabilidad étnica donde se despliega el complejo entramado de relaciones que se cristaliza en los espacios comunitarios que constituyen el centro del fenómeno migratorio. En los mismos se forma la base de la conciencia identitaria a partir de la transmisión de la lengua y otros hábitos culturales (Mera, 2009; Mera, 2011). De manera tal que se da una relación simbólica y material con la tierra de origen que se expresa a partir de una relación tripartita entre la existencia de un anclaje nacional (de residencia), una conciencia nacional (sobre el origen), y una identidad producto del intercambio con otras comunidades. De acuerdo con este enfoque el barrio migrante se establece como una red de producción simbólica en tanto articulación de espacios físicos objetivados y apropiados, fundadores de marcos de pertenencia que aseguran la construcción de la identidad de la diáspora. Identidad que se va a ver atravesada por la articulación con el país de origen y las comunidades migrantes. En el caso de los barrios de migrantes coreanos en todo el mundo estos no van a ser sólo el lugar físico donde se establecen los migrantes en tanto facilitador de su instalación en la sociedad receptora sino "aquel espacio social donde se reproducen las relaciones a través de las cadenas migratorias e intercambios dinámicos múltiples que redefinen en cada etapa el sentido del espacio de pertenencia" (Mera, 2011, p. 7). Es en estos espacios donde se concilia parcialmente el doble problema al que se enfrenta la diáspora coreana tanto en América Latina como en el resto del mundo: por un lado la integración con el país receptor (considerando un modelo de inserción bicultural), y por otro lado la conservación de la identidad trasnacional en un gran número de Estados, sin caer en la segregación con respecto a la sociedad local. En este sentido la concentración espacial en barrios permite la formación de marcos de pertenencia que fortalecen y son condición necesaria para la construcción de la identidad diaspórica. Ahora bien Mera nos advierte que "de ninguna manera debemos pensar que es el amontonamiento lo que la hace una comunidad de diáspora. La unidad reposa sobre los signos, valores y reglas de comportamiento que se producen en los nuevos contextos" (2009, p. 4). Es decir, sobre el ideal de continuidad con el país de origen. De esta manera la noción de diáspora nos es útil para considerar la multiplicidad de elementos articulados relacionalmente que componen a las diversas comunidades coreanas. A la par que nos permite entender la construcción de los barrios migrantes como un aspecto central del fenómeno diaspórico que no podría interpretarse en todas sus dimensiones si lo disociamos del mismo.

A continuación, a partir de las consideraciones hechas en este apartado analizaremos los casos de la inmigración coreana tanto en Buenos Aires como en Guatemala haciendo particular énfasis en la construcción de sus barrios migrantes respectivos: Baek-ku y Korea Town. 


\section{La inmigración coreana en Buenos Aires y la construcción de Baek-ku}

Corea del Sur y la República Argentina establecen relaciones diplomáticas en 1962. Si bien ya hay habitantes provenientes de la península desde 1955, oficialmente la inmigración coreana en Argentina comenzó en octubre de 1965 cuando llega a Buenos Aires el primer barco con migrantes coreanos (el "Boys Ben") que partió desde el puerto de Busan en agosto de ese mismo año (Mera, 2003). Los primeros migrantes se asentarán en Campo Lamarque, en la provincia de Rio Negro. Si bien la mayoría de los migrantes se instalan en Colonias del interior del país, ya en esa época empezarán a establecerse algunas personas aisladamente en la Ciudad de Buenos Aires. Entre 1970 y 1978 llegan al país, aproximadamente 500 familias para establecerse en áreas rurales (Mera, 1998). Finalmente los emprendimientos rurales terminaron fracasando por los escasos recursos económicos con los que contaban los migrantes y el desconocimiento de las prácticas agrícolas sumado a las deficiencias de infraestructura y servicios. Lo que ocasionó que estas familias y las que arribaron en los contingentes sucesivos optaran por instalarse en la Ciudad de Buenos Aires (Bialogorsky, 2004, p. 278). Ya durante los 70's empezarían a asentarse en los barrios de Retiro y de Flores Sur, reforzando la tendencia de permanecer en el ámbito urbano. Además en esa década se empiezan a consolidar los cimientos del grupo étnico-comunitario que se constatará con el crecimiento en los barrios comerciales (Ciudadela, Once y la calle Avellaneda), y la apertura de los comercios (en su mayoría textiles), iglesias y asociaciones (que comenzaron en los 60's). Además, se ponen en circulación, diversos semanarios y periódicos en coreano, entre otros indicadores. En la década del 80 se daría el mayor ingreso de migrantes coreanos, representado por familias provenientes casi en su totalidad de Seúl que arribaron al país por avión. Por lo que no se trata ya de una inmigración con destino rural avalada por el gobierno surcoreano sino "de inmigrantes con capacidad económica para invertir en la pequeña y mediana industria, que arriban estimulados por convenios económicos entre los gobiernos coreanos y argentinos" (Courtis, 2006, p. 133). El principal convenio, firmado en abril de 1985 es el Acta de Procedimiento para el Ingreso de Inmigrantes Coreanos a la Argentina. Entre los años 1984 y 1989 se otorgarán más de 11.000 permisos, lo que representaría el ingreso de más de 20.000 personas. Ya para 1990 lo población originaria de la península de Corea en todo el país alcanzó su punto máximo, contabilizando unas 42000 personas (Mera, 2009). Este período que transcurre entre mediados de la década de 1980 y principios de 1990 puede caracterizarse como la "época de oro de la migración coreana en Argentina” (Mera, 2008, p. 2). Durante el mismo se va producir un crecimiento tanto cualitativo como cuantitativo de las asociaciones, los diarios, las iglesias, etc. Ya durante la década del 90 no se producirían más nuevas olas migratorias sino que se expulsaría población. Cuestión que llegaría a su punto más crítico durante la crisis de 2001 cuando la población pasaría a ser de 15500 habitantes. Posteriormente con la recuperación económica que se dio a partir de 2003 muchos de los miembros de la colectividad retornarían al país, que llega actualmente a los 22000 residentes.

En lo que respecta a la modalidad de inserción de los migrantes coreanos en Buenos Aires, la tendencia predominante fue a concentrarse en unos pocos barrios. En principio se establecieron en villas de emergencia como las de Retiro y luego en barrios como Villa Soldati, Villegas o Ciudadela para finalmente llegar a Flores Sur (también conocido como 
bajo Flores). Es en este barrio donde se va a reunir la principal concentración de coreanos en Buenos Aires hasta el día de hoy. El barrio coreano de Buenos Aires o Baek-ku, que significa 109 en coreano en alusión al número de bus que conectaba el barrio de Retiro con el Bajo Flores, se fue consolidando a la par que los migrantes coreanos lograban insertarse en la producción textil. Tengamos en consideración que Flores es un barrio periférico con respecto al centro de la ciudad y a los centros comerciales de otros barrios de Buenos Aires. Es un barrio modesto y simple, habitado por sectores medios, bajos y populares. Se sitúa en el límite de una villa de emergencia donde residen otros inmigrantes, en general de países limítrofes. Baek-ku en particular, se concentra a lo largo de la avenida Carabobo (que cuadras más tarde pasa a llamarse Corea ${ }^{2}$ ) desde avenida Eva Perón hasta Castañares y hacia los alrededores. En calles cercanas se instalan las residencias particulares, al igual que en otros barrios como Caballito y Balvanera (donde se encuentra la zona de comercial de "Once"). En Caballito, a lo largo de la avenida Avellaneda, se encuentran comercios textiles bolivianos orientados a los consumidores argentinos manejados por judíos, coreanos. En el de "Once" también se localizaron los primeros comercios coreanos de venta textil, en competencia con los tradicionales negocios de judíos (Sassone y Mera, 2007). Es importante considerar que la introducción de los migrantes en el rubro textil se dio de la mano de los dueños argentinos de origen judío que derivaron parte del proceso de producción hacia las familias coreanas que realizaban el trabajo en sus propias viviendas. Bialogorsky plantea que los 3 factores principales que explican el crecimiento de esta actividad entre los residentes coreanos fueron que "la empresa sea originariamente familiar, una cultura del trabajo basada en largas jornadas laborales y la utilización de redes comunitarias con el gae" (2004, p. 284). El gae o kye es un tipo de cooperación informal que consiste en un sistema de crédito rotativo que se da dentro de la red solidaria comunitaria. El mismo consiste en que un conjunto de familias se reúne y deposita una vez por mes una determinada cantidad de dinero que luego será sorteada; permitiéndoles a algunos de los participantes invertir dichas sumas en sus emprendimientos.

En el barrio se encuentran toda clase de locales comerciales, los cuales en su gran mayoría tienen carteles e inscripciones en coreano: panaderías con productos típicos, casas de modas, salones de belleza, peluquerías, dentistas, garajes, talleres mecánicos, supermercados con productos con embalajes coreanos, casas de arroz, restaurantes que en general no están a la vista del pasante sino ubicados al final de largos pasillos, pescaderías, agencias inmobiliarias, casas de computación, de regalos, el Golf Shop, la Confederación Argentina de Tae Kwon Do, estudios contables, una farmacia, compañías de remises, agencia de viaje y turismo, etc. (Mera, 1998). Todos estos comercios y servicios se encuentran orientados a la comunidad coreana. El hecho de que los menús, carteles de publicidad y hasta los precios estén escritos en hangul (lengua coreana) nos da la pauta de que no hay interés en captar más interesados que los de la propia comunidad sino más bien que todo está preparado para el consumo étnico-comunitario (Mera, 2005). Durante la década del ochenta, cuando la comunidad se estaba expandiendo, la concentración de locales étnicos generó un fuerte rechazo entre los habitantes de Flores. A esto se le sumaba que en la mayoría de los casos los vendedores de los negocios no hablan español, los productos no están preparados para la venta y consumo de los argentinos, (pescado seco, algas de mar, nabos gigantes y otras verduras aún poco conocidas Argentina) o simplemente porque a 
causa del tipo de embalaje resulta casi imposible identificar el contenido. Esto generó en principio una convivencia algo complicada con episodios de discriminación y ataques a la comunidad por parte de los medios de comunicación ${ }^{3}$. Cuestiones que por suerte en la actualidad ya no tienen ni por asomo el nivel de agresividad de los 80's y 90's.

Más allá de estos episodios existen en el barrio una gran cantidad de espacios que permiten desarrollar una vida asociativa intensa. Centralmente son o espacios recreativos como bares y cantobares e institucionales-religiosas como las iglesias y asociaciones. En el barrio las iglesias se encargan de realizar la conexión entre los individuos de los diferentes espacios de la comunidad. Hay varias iglesias evangelistas, una católica y dos templos budistas. En cuanto a las demás instituciones están pueden englobarse dentro de asociaciones, medios de comunicación (diarios y radio) y comercios varios, entre otros. Además de la Asociación Coreana en Argentina (fundada en 1966) existen otras de carácter muy variado: por origen de provincia, por universidades y escuelas de graduación en Corea, por actividades laborales desarrolladas en Corea antes de emigrar, deportivas, artísticas y de escritores (Mera, 1998). Hay, asimismo, asociaciones relacionadas con las distintas opciones de vida en la ciudad de Buenos Aires: de comerciantes, de industriales, de profesionales (médicos, abogados, contadores, etc.) de estudiantes, de jugadores de golf y de ancianos. Entre las mismas también se encuentran el Club de calígrafos, el Grupo de estudio de la cultura de los inmigrantes coreanos, el Grupo de Bellas Artes y las iglesias cristianas (Sassone y Mera, 2007). Esta amplia gama de instituciones juega un rol muy importante en la forma en que las personas ordenan su vida cotidiana y contribuye fuertemente al desarrollo de la vida de la comunidad en nuestro país (Mera, 2005).

La formación de Baek-ku "fortificó la cohesión del grupo al mismo tiempo que marcó de manera muy particular el tipo de diálogo establecido con otro grupos migrantes y con la sociedad receptora" (Mera, 2008, p. 3). A partir de esto se desprenden tres aspectos centrales que hacen a la caracterización del barrio coreano de Buenos Aires. El primero es su consolidación el tiempo. Baek-ku, al igual que la comunidad ya tienen más de 50 años de historia. El segundo es el reconocimiento de su representatividad por parte de la propia comunidad. Lo cual se puede apreciar al observar la importante vida asociativa cultural, comercial y religiosa que tienen los miembros de la comunidad en el barrio y que le dan su impronta étnica. A lo que se le suma además una función residencial del barrio. Y el tercero refiere a las marcas étnicas diferenciales en el paisaje urbano. No sólo ha estado mucho tiempo, y es reconocido por los propios residentes coreanos sino que los propios no coreanos reconocen también que ese espacio social comprendido en los límites de la Avenida Carabobo es algo particular.

\section{La inmigración coreana en Guatemala y la construcción de Korea Town}

Si bien Guatemala y la República de Corea establecieron formalmente relaciones diplomáticas en 1962 no sería hasta mediados de los 80's que empezarían a llegar los primeros migrantes surcoreanos a ese país. Más precisamente a partir de 1985, 20 años después que Argentina. En este caso no eran ni migrantes rurales (o al menos traídos con la finalidad 
de participar en colonias agrícolas) ni tampoco sectores profesionales y medios que encontraban demasiadas trabas para ascender en la competitiva sociedad surcoreana. En Guatemala los primeros coreanos que llegaron eran empleados de importantes corporaciones surcoreanas (Jin, 2017, p. 76). Muchas de estas empresas, preponderantemente orientadas al sector textil empezaron a instalarse en Centroamérica durante la década del 80. Esto se debió a que por un lado a que las grandes empresas surcoreanas se encontraban en un proceso de marcada transnacionalización y por otro a que la mano de obra barata que permitió el crecimiento exponencial de la industria textil surcoreana en los 70's (al punto de ser una de las principales ramas de su economía) ya no era tal. Joo Ok Kim destaca que durante los 80's se llevaron a cabo una serie de huelgas (que se acoplaron también a los movimientos pro democratización) que tuvieron mucha fuerza en el sector textil; logrando arrancar a las patronales toda una serie de reivindicaciones sin precedentes hasta ese momento para la clase obrera surcoreana, entre ellos la conquista del convenio colectivo (2017, p. 196). A lo que se le suma también la instauración del primer salario mínimo en 1987. Como respuesta a esto las grandes empresas surcoreanas del sector textil mudaron gran parte de su producción a Centroamérica. Esto implicó una importante salida de personal coreano que incluía empleados jerárquicos, gerentes, técnicos y otros trabajadores calificados (Jin, 2017, p. 76). Entre las principales alternativas para invertir, escapando de los altos salarios y el activismo creciente en Corea del Sur, estas empresas hallaron en la Guatemala rural una gran masa de fuerza de trabajo (en su mayoría de la etnia Maya), ansiosa por conseguir empleos en una industria "moderna" que les permita expandir sus oportunidades (Goldin, 2012, p. 25). Pues bien, lamentablemente lo que consiguieron estos trabajadores (en su gran mayoría mujeres jóvenes) fueron jornadas extenuantes, salarios muy bajos y nulos derechos laborales. Plantea Kim que las maquiladoras pertenecientes a los grandes conglomerados surcoreanos que operaban en Guatemala tenían métodos para extraer productividad de los trabajadores que iban más allá de la "norma" de explotación (2017, p. 200). Entre 1988 y 1991 operaban en Guatemala cerca de 50 maquiladores con propietarios surcoreanos reportando ganancias aproximadas de 150 millones de dólares por año (Petersen, 1992). A diferencia de Argentina y Brasil en donde la producción textil estaba orientada al consumo interno, en el caso de las maquiladoras la orientación es plenamente hacia la exportación, siendo Estados Unidos el principal destino.

En lo que refiere a la Comunidad coreana de Guatemala, casi la totalidad de sus miembros viven en la Capital. Se destacan las actividades de la Asociación de Coreanos en Guatemala, la Asociación Coreana de la Industria Textil y la apertura de un Colegio Coreano en 1993 que también alberga un centro cultural desde 2016. Además se resalta la existencia de restaurantes, cantobares, clínicas, mini supermercados e iglesias. En 2013 anunció la realización de un proyecto urbanístico bautizado como Korea Town de participación tripartita involucrando a la Asociación de Coreanos en Guatemala, la embajada de la República de Corea y la Municipalidad de Guatemala. El mismo se localiza en la partir de la zona 7 entre la Avenida Seúl (oficializada el 9 de abril de 2014), la calzada Mateo Flores y la calle 1, en la Ciudad de Guatemala.

Entre los principales objetivos del proyecto se destacan la mejora de la seguridad y la infraestructura urbana; la promoción de la economía y los recursos turísticos del área; la 
promoción de oportunidades de inversión desde Corea; y la contribución al acercamiento y mutuo entendimiento de ambas culturas. La primera parte del proyecto se inauguró el 14 de Mayo de 2014. Las inversiones iniciales estuvieron destinadas a la instalación de un circuito cerrado de vigilancia, 30 lámparas con estilo coreano que se alimentarán con energía solar y un radar de velocidad vehicular ${ }^{4}$. Actualmente Korea Town está orientada al turismo y cuenta con una gran cantidad de negocios de diversa índole. Lo que no quita que tenga además una función cultural y residencial, ya que tanto el centro cultural abierto en 2016 como gran parte de las residencias de los miembros de la comunidad se encuentran en la zona 7 de la ciudad, que es donde está emplazado Korea Town.

\section{Contrapunto y Conclusiones}

A partir del análisis realizado concluimos que los barrios étnicos emergen en América Latina evidenciando procesos globales y locales. En este sentido, tanto Korea Town como Baek-ku, podrían ser considerados como dos modelos distintos de construcción de relaciones de identidad étnicas. En ambos casos se comparte la doble tensión que surge de la inserción en la sociedad local por un lado y la conservación de la identidad diaspórica por otro. Aun así la articulación con las instituciones y actores de los espacios locales son diferentes. Mientras que en Argentina Baek-ku se sitúa en un barrio periférico en un contexto aislamiento geográfico parcial (que en este caso no es acompañado de un proceso de pauperización de sus miembros) Korea Town es un proyecto urbanístico que cuenta con apoyo estatal y se encuentra orientado al turismo. Esto implica que la comunidad coreana de Guatemala debe aunar sus esfuerzos para fortalecer los espacios interculturales entre migrantes y locales para lograr un mayor diálogo entre los distintos actores. En este sentido vemos que cada comunidad coreana en la región pudo, por un lado desarrollarse en acuerdo a la cultura local, pero por el otro, mantuvo sus tradiciones; organizándose en torno a las necesidades de la colectividad.

El rol de las instituciones (iglesias, templos y asociaciones) que fueron mediadoras de la cultura local y la coreana fue central para la aceptación e integración de las comunidades en la región. Cultivar el diálogo intercultural con ellas y desarrollar categorías interpretativas sólidas para abordar los fenómenos migratorios en su complejidad desde una óptica latinoamericana son tareas pendientes que vemos desprenderse de lo que aquí desarrollamos.

\section{Notas}

1. Serie histórica de población migrante desde 2001 en adelante disponible en: http:// www.index.go.kr/potal/main/EachDtlPageDetail.do?idx_cd=1682

2. Renombrada así en 1989 en reconocimiento al barrio que comenzó a formarse con las llegada de migrantes a mediados de la década de 1960. 
3. Escapa a los fines de este trabajo pero a modo ilustrativo citaré algunos titulares de artículos periodísticos con impronta claramente discriminatoria: "Se comprobó que hay coreanos explotadores.

"La esclavitud que llegó de Oriente se quedó en Flores Sur" (La Nación, del 21 de abril de 1993); "Instalarán medidores de luz en las villas porteñas. Los villeros pagarán, ¿y los coreanos? En el bajo Flores los coreanos también roban la electricidad" (La Nación, del 14 de julio de 1993).

4. Korea Town en Guatemala busca ser foco turístico. (Publinews 13 de Mayo de 2014). Recuperado de: https://www.publinews.gt/gt/tendencias/2014/05/13/korea-town-guatemalabusca-foco-turistico.html

\section{Referencias bibliográficas}

Bialogorsky, M. (1996). La presencia coreana en la Argentina: La construcción simbólica de una experiencia migratoria. Tesis de doctorado de la Universidad de Buenos Aires. Recuperada de http://www.centro-de-semiotica.com.ar/BialogorskiTesis.pdf

Bialogorsky, M. (2004). Argentina. Logros de una inmigración reciente. En: Cuando Oriente Llegó a América. Washington D.C. BID.

Courtis,C. (2000). Construcción de Alteridades: Discursos sobre la inmigración coreana en Buenos Aires. Buenos Aires, Argentina: EUDEBA.

Courtis, C. (2006). Una aproximación lingüistico-antropológica a la inmigración coreana en Buenos Aires. Migraciones contemporáneas y diversidad cultural en la Argentina. Córdoba; p. 131-154.

Goldin, L. (2012). From Despair to Resistance: Maya Workers in the Maquilas of Guatemala. Anthropology of Work Review. Volume XXXIII, Number 1.

Jin, S. (2017). Arriving with Machines, Skills, and Transnational Networks: Korean Immigrants' Involvement in the Garment Industry in Latin America OMNES: The Journal of Multicultural Society 2017. Vol.8 No.1, pp. 64-84.

Kim, J. (2017). “Training Guatemalan Campesinos to Work Like Korean Peasants”: Taxonomies and Temporalities of East Asian Labor Management in Latin America Verge: Studies in Global Asias, Vol. 3, No. 2, Between Asia and Latin America: New Transpacific Perspectives (Fall 2017), pp. 195-216.

Mera, C. (1998). La inmigración coreana en Buenos Aires. Multiculturalismo en el espacio urbano. Buenos Aires: EUDEBA.

Mera, C. (2003). La inmigración coreana en Buenos Aires. Historia y actualidad. Ponencia presentada en el XI Congreso Internacional de ALADAA. 12 a 15 de Noviembre de 2003. Ciudad de México.

Mera, C. (2005). "Modalidad de instalación de la comunidad coreana en la Ciudad de Buenos Aires”, en Mera C.; Cosiansi, L. y Gonzalez, C. Coreanos en Argentina: 40 años de Historia. Buenos Aires: Editorial Al Margen.

Mera, C. (2008). La comunidad coreana de Buenos Aires: una experiencia de convivencia intercultural. En: Sociedad Vol. 27 p. 83-93. 
Mera, C. (2009). "La diáspora coreana en América Latina” en Ramirez Bonilla, J. Transiciones coreanas: permanencia y cambio en Corea del Sur en el inicio del siglo XXI. México: Colegio de México.

Mera, C. (2011). El concepto de diáspora en los estudios migratorios: reflexiones sobre el caso de las comunidades y movilidades coreanas en el mundo actual. En Revista de Historia $n^{\circ}$ 12, Facultad Nacional de Humanidades. Universidad Nacional del Comahue. Petersen, K. (1992). "Zones of Exploitation: Korean Investment on Guatemala", Multinational Monitor. Recuperado de: https://multinationalmonitor.org/hyper/issues/1992/12/ mm1292_10.html

Radulovich, R. (2015). ¿Cómo se produjo la inmigración coreana en Argentina y cuáles fueron las diferencias con otros países de América Latina? en Paula Iadevito y Luciano Lanare (comp) Estudios Coreanos en el escenario Sur-Sur. Buenos Aires: Ed. Imago Mundi. Sassone, S. y Mera, C. (2007). Barrios de migrantes, espacios interculturales: coreanos y bolivianos en la Ciudad de Buenos Aires. XXVI Congreso de la Asociación Latinoamericana de Sociología. Asociación Latinoamericana de Sociología, Guadalajara.

Abstract: In this article we propose a historical-comparative analysis of Korean immigration to Latin America based on the cases of Argentina and Guatemala; focusing on community constitution processes that is expressed in the construction of migrant neighborhoods. In this sense we will identify and describe what were the main factors that influenced the settlement of the first korean migrants and the conformation of their communities; and also which are the dynamics that currently go through them both in Buenos Aires and Guatemala City.

Keywords: International migrations - diaspora - immigrant neighborhoods - Korea Latin America.

Resumo: Neste artigo, propomos realizar uma análise histórico-comparativa da imigração coreana para a América Latina a partir dos casos da Argentina e da Guatemala; tomando como eixo transversal destes processos de constituição comunitária, a construção de bairros migrantes. Dessa forma, procuraremos identificar e descrever quais foram os principais fatores que afetaram o assentamento dos primeiros migrantes coreanos e a formação das comunidades; e também quais são as dinâmicas que estão passando por eles em Buenos Aires e na cidade da Guatemala.

Palavras chave: migrações internacionais - bairros de migrantes - diáspora - Coréia América Latina.

[Las traducciones de los abstracts fueron supervisadas por el autor de cada artículo] 